\title{
Correspondence
}

\section{The Incidence and Timing of Blood Cultures in Multiple Myeloma - Results from a Retrospective, Single Center, Real-World Study}

\author{
Agoston Gyula Szabo ${ }^{1,2, *}, \mathbb{D}$, Katrine Fladeland Iversen ${ }^{1,2,(\mathbb{D}}$, Flemming Schønning Rosenvinge ${ }^{3,(\mathbb{D})}$ \\ Sören Möller ${ }^{2,4,(D)}$, Torben Plesner ${ }^{1,2}$ \\ ${ }^{1}$ Department of Hematology, Vejle Hospital, Beriderbakken 4, Vejle 7100, Denmark \\ ${ }^{2}$ University of Southern Denmark, J.B. Winsløws Vej 9 A, Odense C 5000, Denmark \\ ${ }^{3}$ Department of Clinical Microbiology, Odense University Hospital, J.B. Winsløws Vej 4, Odense C 5000, Denmark \\ ${ }^{4}$ OPEN - Open Patient data Explorative Network, Odense University Hospital and Department of Clinical Research, Odense C 5000, Denmark
}

\author{
ARTICLE INFO \\ Article History \\ Received 14 May 2020 \\ Accepted 09 July 2020 \\ Keywords \\ Blood cultures \\ multiple myeloma \\ infection \\ incidence \\ risk factor
}

(C) 2020 International Academy for Clinical Hematology. Publishing services by Atlantis Press International B.V. This is an open access article distributed under the CC BY-NC 4.0 license (http://creativecommons.org/licenses/by-nc/4.0/)

\section{INTRODUCTION}

Multiple myeloma (MM) is a cancer of the immune system characterized by complex cellular and humoral immunodeficiency [1-3]. Patients with MM often have advanced age, increased comorbidity and are exposed to immunosuppressive drugs during therapy [4]. These factors contribute to markedly increased risk of infections, mainly pneumonia and sepsis [5-7]. These complications are responsible for a substantial part of early deaths in MM [7-9]. The incidence of bacterial infections peaks in the first six months after diagnosis $[10,11]$. We have previously reported the results of a retrospective, single-center, real-world study conducted at Vejle Hospital (a primary and secondary referral center receiving approximately 30 newly diagnosed cases of MM per year), in which we reviewed the clinical course of 303 patients with MM who initiated treatment from 2006 to 2016 [12]. The aims of the present study were to describe, in a single-center cohort of patients with MM, the results of blood cultures, to determine their incidence and timing, and to assess their associations with baseline clinical characteristics and treatment-related factors. Moreover, we assessed the use of immunoglobulin replacement therapy (IGRT) in the study population.

We collected blood culture results from the Danish microbiology register (MiBA [13]) of all hospitals where patients were treated. A 'blood culture day' (BCD) was defined as a day a patient had at least

"Corresponding author. Email: agoston.gyula.szabo@rsyd.dk

Peer review under responsibility of the International Academy for Clinical Hematology one blood culture performed. A positive BCD was defined as a day a patient had at least one positive blood culture result. Blood cultures were categorized as "negative", "positive" or "possible contamination" according to the guidelines for classification of hospital-acquired blood stream infections in the Danish Healthcare-associated Infections Database [14]. Blood cultures classified initially as "possible contamination" were reclassified as blood stream infections if the same microorganism was identified in the patient's blood cultures more than once in a period of 14 days. We assessed the time to first blood culture and first positive blood culture by the Kaplan-Meier method. We calculated BCDs over time as counts per 1000 patients. The number of BCDs was described with median, range and interquartile range (IQR). We investigated univariate and multivariate association of baseline patient characteristics and treatment-related factors, reported as hazard ratios with $95 \%$ confidence intervals, with time to BCDs by Cox regression. We included repeated events in the Cox regression model to take into account multiple blood cultures for the same patient. Blood culture data were available in 302 patients. Two hundred and eighty-two patients had at least one blood culture result and 113 patients had at least one positive blood culture result. We identified 4992 blood culture results: 4243 were negative and 249 were positive. Fifty-nine positive blood culture results were classified as possible contaminations. The most frequently cultured pathogenic microorganisms were Enterobacterales (31\%; of these $75 \%$ Escherichia coli), Streptococcus pneumoniae (17\%) and Coagulasenegative Staphylococci (11\%). The full list of cultured microorganisms is shown in Table S1. The median number of blood cultures 
per patient was six (IQR: 3; 11; range: $0 ; 43$ ). The incidence of BCDs peaked in the month of diagnosis ( $>500 / 1000$ patients/month) and was elevated in the first 6 months after diagnosis (Figure 1A). After this period, the incidence of BCDs was constantly below 200/1000 patients/month. Fifty-one percent, $67 \%$ and $74 \%$ of patients had a BCD 3, 6 and 12 months after diagnosis, respectively (Figure 1B). Besides the time of diagnosis, we found incidence peaks in BCDs in relation to initiation of both the first and later lines of therapy (>600/1000/month) and events of progressive disease (>700/1000/ month), as shown in Figure 2.

Univariate and multivariate analyses of risk factors for blood culture days are shown in Table S2. Among clinical baseline characteristics, low hemoglobin, high ionized calcium, high creatinine, low immunoglobulin (Ig) M, M-protein of IgA isotype, light-chain only disease and poor Eastern Cooperative Oncology Group (ECOG) performance status were independently associated with higher risk of BCDs. Among treatment-related factors, worse than very good partial response to the ongoing line of therapy, increasing number of prior lines of therapy and a recent event of progressive disease were independently associated with higher risk of BCDs. Among the assessed treatment regimens, high-dose melphalan (days 0-30), proteasome inhibitor-steroid doublets, chemotherapy-steroid doublets, daratumumab monotherapy and intensive combination regimens including five or more drugs were independently associated with such higher risk of BCDs. Two hundred and nine (69\%) patients were exposed to immunoglobulin replacement therapy with a median time of 3.2 (IQR: 0.9; 22.3) months after diagnosis.
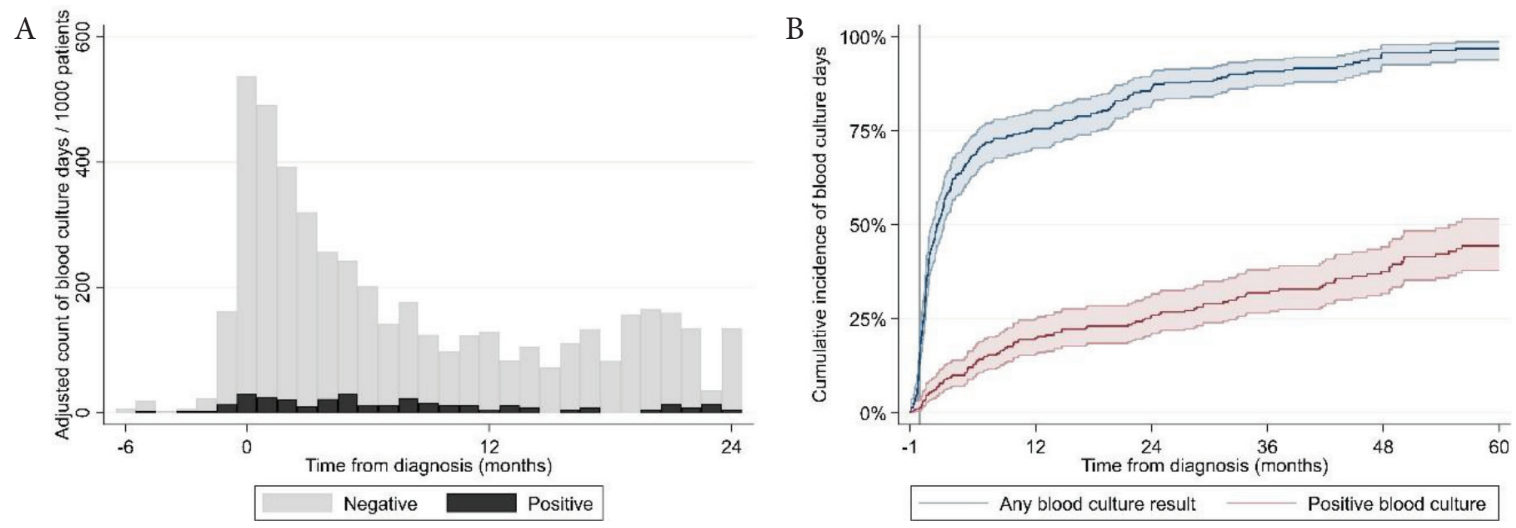

Figure 1 (A) Adjusted incidence of blood culture positivity from the time of diagnosis. (B) Cumulative incidence of blood culture positivity from the month prior to diagnosis until death or follow up at 60 months.
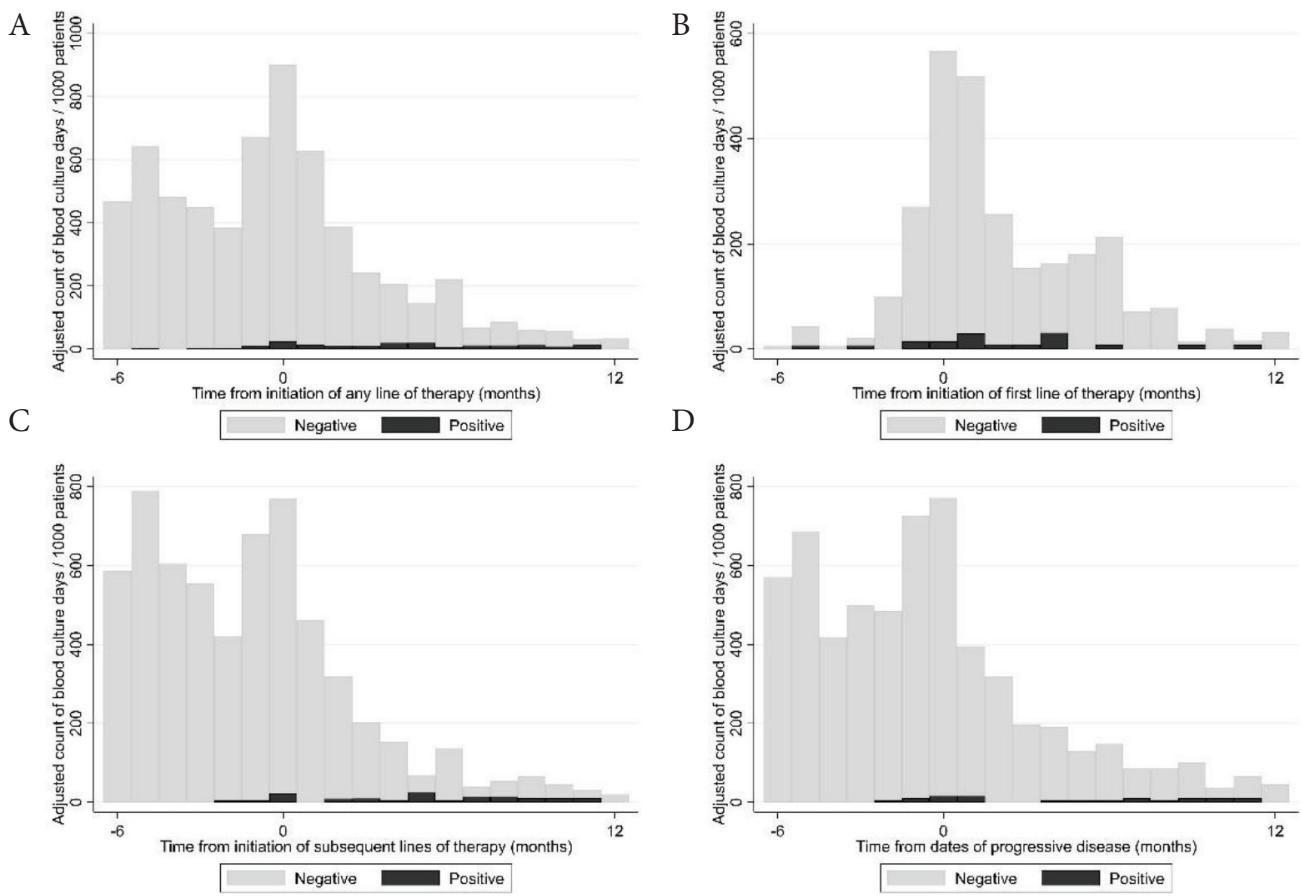

Figure 2 (A) Adjusted incidence of blood culture days in relation to initiation of any line of therapy. (B) Adjusted incidence of blood culture days in relation to the date of initiation of the first line of therapy. (C) Adjusted incidence of blood culture days in relation to the date of initiation of any subsequent line of therapy. (D) Adjusted incidence of blood culture days in relation to the dates of progressive disease. 
In conclusion, the burden of infections in our cohort of MM patients was high. Blood stream infections were mainly caused by Gram-negative bacteria. Patients were especially susceptible to infections at the time of diagnosis and in situations of insufficient disease control. Certain anti-myeloma regimens, such as proteasome inhibitor-steroid doublets, chemotherapy-steroid doublets, daratumumab monotherapy and combination regimens including five or more drugs may require increased attention to infectious complications.

\section{CONFLICTS OF INTEREST}

AGS: consulting for Janssen; TP: consulting for Janssen, Celgene, Takeda, Abbvie, Genmab; KFI: no conflicts of interest; SM: no conflicts of interest; FSR: no conflicts of interest.

\section{AUTHORS' CONTRIBUTION}

AGS designed the study, created the study database, conducted patient chart review, wrote the manuscript and designed the figures. KFI conducted patient chart review and contributed to writing the manuscript. SM carried out data analysis and statistics and contributed to writing the manuscript. FSR reviewed and categorized the blood culture results and contributed to writing the manuscript. TP supervised the study, contributed to writing the manuscript and designing the figures.

\section{FUNDING}

The authors received no financial support for the research, authorship or publication of this article.

\section{ACKNOWLEDGMENTS}

We thank the Department of Internal Medicine and the Hematological Clinical Research Unit at Vejle Hospital for providing the financial and logistical background for this study. Data management for this project was carried out by Open Patient Data Exploratory Network, University of Southern Denmark.

\section{REFERENCES}

[1] Pratt G, Goodyear O, Moss P. Immunodeficiency and immunotherapy in multiple myeloma. Br J Haematol 2007;138;563-79.
[2] Kastritis E, Zagouri F, Symeonidis A, Roussou M, Sioni A, Pouli A, et al. Preserved levels of uninvolved immunoglobulins are independently associated with favorable outcome in patients with symptomatic multiple myeloma. Leukemia 2014;28;2075-9.

[3] Heaney JLJ, Campbell JP, Iqbal G, Cairns D, Richter A, Child JA, et al. Characterisation of immunoparesis in newly diagnosed myeloma and its impact on progression-free and overall survival in both old and recent myeloma trials. Leukemia 2018;32;1727-38.

[4] Gregersen H, Vangsted AJ, Abildgaard N, Andersen NF, Pedersen RS, Frølund UC, et al. The impact of comorbidity on mortality in multiple myeloma: a Danish nationwide population-based study. Cancer Med 2017;6;1807-16.

[5] Schütt P, Brandhorst D, Stellberg W, Poser M, Ebeling P, Müller $S$, et al. Immune parameters in multiple myeloma patients: influence of treatment and correlation with opportunistic infections. Leuk Lymphoma 2006;47;1570-82.

[6] Nucci M, Anaissie E. Infections in patients with multiple myeloma in the era of high-dose therapy and novel agents. Clin Infect Dis 2009;49;1211-25.

[7] Blimark C, Holmberg E, Mellqvist UH, Landgren O, Björkholm M, Hultcrantz M, et al. Multiple myeloma and infections: a population-based study on 9253 multiple myeloma patients. Haematologica 2015;100;107-13.

[8] Augustson BM, Begum G, Dunn JA, Barth NJ, Davies F, Morgan G, et al. Early mortality after diagnosis of multiple myeloma: analysis of patients entered onto the United kingdom Medical Research Council trials between 1980 and 2002-Medical Research Council Adult Leukaemia Working Party. J Clin Oncol 2005;23;9219-26.

[9] Holmström MO, Gimsing P, Abildgaard N, Andersen NF, Helleberg C, Clausen NAT, et al. Causes of early death in multiple myeloma patients who are ineligible for high-dose therapy with hematopoietic stem cell support: a study based on the nationwide Danish Myeloma Database. Am J Hematol 2015;90;E73-E4.

[10] Teh BW, Harrison SJ, Worth LJ, Spelman T, Thursky KA, Slavin MA. Risks, severity and timing of infections in patients with multiple myeloma: a longitudinal cohort study in the era of immunomodulatory drug therapy. Br J Haematol 2015;171;100-8.

[11] Sørrig R, Klausen TW, Salomo M, Vangsted A, Gimsing P. Risk factors for blood stream infections in multiple myeloma: a population-based study of 1154 patients in Denmark. Eur J Haematol 2018;101;21-7.

[12] Szabo AG, Iversen KF, Möller S, Plesner T. The clinical course of multiple myeloma in the era of novel agents: a retrospective, single-center, real-world study. Clin Hematol Int 2019;1;220-8.

[13] MiBa, the Danish Microbiology Database. Available from: https:// miba.ssi.dk/service/english.

[14] HAIBA - Bakteriæmi. Available from: https://miba.ssi.dk/haiba/ casedefinitioner/bakteriaemi. 


\section{SUPPLEMENTARY MATERIALS}

Table S1 Microorganisms cultured on positive blood culture days

\begin{tabular}{lcc}
\hline Pathogenic microorganism & $\boldsymbol{n}$ & Percentage (\%) \\
\hline Enterobacterales $^{\star}$ & 59 & 31 \\
Streptococcus pneumoniae & 33 & 17 \\
Coagulase-negative Staphylococci & 21 & 11 \\
Staphylococcus aureus & 14 & 7 \\
Anaerobic bacteria & 14 & 7 \\
Enterococci & 13 & 7 \\
Viridans streptococci & 7 & 4 \\
Listeria monocytogenes & 6 & 3 \\
Pseudomonas aeruginosa & 6 & 3 \\
Candida & 6 & 3 \\
Beta-hemolytic streptococci & 6 & 3 \\
Other bacteria & 5 & 3 \\
Total (pathogenic microorganisms) & 190 & \\
Possible contaminations & 59 & \\
Total (positive) & 249 & \\
\hline
\end{tabular}

"44 (75\%) Escherichia coli. A positive BCD was defined as a day a patient had at least one positive blood culture result.

Table S2 Univariate and multivariate analysis of risk factors for blood culture days

\begin{tabular}{|c|c|c|c|c|c|}
\hline \multirow{2}{*}{ Variable } & \multirow{2}{*}{$\begin{array}{l}\text { Number of } \\
\text { observations }\end{array}$} & \multirow{2}{*}{$\begin{array}{c}\text { Univariate analysis } \\
\text { HR }(95 \% \mathrm{CI})\end{array}$} & \multirow{2}{*}{$p$} & \multirow{2}{*}{$\frac{\text { Multivariate analysis }}{\text { HR }(95 \% \mathrm{CI})}$} & \multirow{2}{*}{$p$} \\
\hline & & & & & \\
\hline \multicolumn{6}{|l|}{ Demographics } \\
\hline \multicolumn{6}{|l|}{ Age } \\
\hline$<50$ & 14 & Reference & & Reference & \\
\hline $50-60$ & 36 & $0.94(0.77 ; 1.15)$ & 0.567 & $0.67(0.53 ; 0.85)$ & 0.001 \\
\hline $60-70$ & 106 & $1.07(0.90 ; 1.28)$ & 0.434 & $0.67(0.54 ; 0.83)$ & $<0.001$ \\
\hline$>70$ & 146 & $1.01(0.84 ; 1.21)$ & 0.939 & $0.61(0.49 ; 0.75)$ & $<0.001$ \\
\hline \multicolumn{6}{|l|}{ Sex } \\
\hline Male & 175 & Reference & & Reference & \\
\hline Female & 127 & $0.85(0.79 ; 0.93)$ & $<0.001$ & $0.93(0.85 ; 1.03)$ & 0.154 \\
\hline \multicolumn{6}{|l|}{ CRAB features } \\
\hline Hemoglobin $<6.2 \mathrm{mmol} / \mathrm{L}$ & 102 & $0.85(0.82 ; 0.88)$ & $<0.001$ & $0.91(0.86 ; 0.95)$ & $<0.001$ \\
\hline Ionized calcium $>1.345 \mathrm{mmol} / \mathrm{L}$ & 79 & $1.65(1.36 ; 2.01)$ & $<0.001$ & $1.38(1.09 ; 1.76)$ & 0.008 \\
\hline Creatinine $>177 \mu \mathrm{mol} / \mathrm{L}$ & 57 & $1.00(1.00 ; 1.00)$ & $<0.001$ & $1.00(1.00 ; 1.00)$ & 0.005 \\
\hline Osteolytic lesion on either skeletal X-ray or WBLDCT not present & 97 & Reference & & Reference & \\
\hline Osteolytic lesion on either skeletal X-ray or WBLDCT present & 205 & $0.85(0.78 ; 0.93)$ & $<0.001$ & $0.75(0.68 ; 0.83)$ & $<0.001$ \\
\hline \multicolumn{6}{|l|}{ Immunoglobulins } \\
\hline $\operatorname{IgA} \geq 0.7 \mathrm{~g} / \mathrm{L}$ (excluding patients with IgA M-protein isotype) & 133 & Reference & & Reference & \\
\hline IgA $<0.7 \mathrm{~g} / \mathrm{L}$ (excluding patients with IgA M-protein isotype) & 169 & $1.05(0.97 ; 1.14)$ & 0.213 & $0.91(0.80 ; 1.04)$ & 0.180 \\
\hline IgG $\geq 6.1 \mathrm{~g} / \mathrm{L}$ (excluding patients with IgG M-protein isotype) & 190 & Reference & & Reference & \\
\hline IgG $<6.1 \mathrm{~g} / \mathrm{L}$ (excluding patients with IgG M-protein isotype) & 112 & $1.33(1.23 ; 1.45)$ & $<0.001$ & $0.61(0.49 ; 0.75)$ & $<0.001$ \\
\hline $\operatorname{IgM} \geq 0.4 \mathrm{~g} / \mathrm{L}$ & 50 & Reference & & Reference & \\
\hline $\operatorname{IgM}<0.4 \mathrm{~g} / \mathrm{L}$ & 252 & $1.90(1.67 ; 2.17)$ & $<0.001$ & $1.77(1.48 ; 2.11)$ & $<0.001$ \\
\hline \multicolumn{6}{|l|}{ M-protein isotype } \\
\hline IgA & 59 & $1.44(1.30 ; 1.60)$ & $<0.001$ & $1.52(1.18 ; 1.94)$ & 0.001 \\
\hline IgG & 168 & Reference & & Reference & \\
\hline Light-chain only & 54 & $1.02(0.91 ; 1.14)$ & 0.789 & $1.37(1.11 ; 1.69)$ & 0.004 \\
\hline Non-secretory & 6 & $0.76(0.55 ; 1.04)$ & 0.088 & $1.06(0.72 ; 1.56)$ & 0.779 \\
\hline Other & 2 & $2.71(1.68 ; 4.39)$ & $<0.001$ & $4.15(2.34 ; 7.34)$ & $<0.001$ \\
\hline \multicolumn{6}{|l|}{ Myeloma risk profile } \\
\hline ISS I & 88 & Reference & & Reference & \\
\hline ISS II & 92 & $1.11(1.00 ; 1.23)$ & 0.043 & $1.01(0.89 ; 1.14)$ & 0.848 \\
\hline ISS III & 72 & $1.55(1.39 ; 1.73)$ & $<0.001$ & $0.87(0.75 ; 1.01)$ & 0.076 \\
\hline
\end{tabular}


Table S2 Univariate and multivariate analysis of risk factors for blood culture days-Continued

\begin{tabular}{|c|c|c|c|c|c|}
\hline \multirow{2}{*}{ Variable } & \multirow{2}{*}{$\begin{array}{c}\text { Number of } \\
\text { observations }\end{array}$} & \multirow{2}{*}{$\begin{array}{c}\text { Univariate analysis } \\
\text { HR (95\% CI) }\end{array}$} & \multirow{2}{*}{$p$} & \multirow{2}{*}{$\frac{\text { Multivariate analysis }}{\operatorname{HR}(95 \% \mathrm{CI})}$} & \multirow{2}{*}{$p$} \\
\hline & & & & & \\
\hline ISS not determined & 47 & $1.16(1.02 ; 1.32)$ & 0.024 & $0.92(0.79 ; 1.08)$ & 0.300 \\
\hline High risk cytogenetics $[\mathrm{t}(4 ; 14), \mathrm{t}(14 ; 16)$ or $\operatorname{del}(17 \mathrm{p})]$ by FISH not present & 254 & Reference & & Reference & \\
\hline High risk cytogenetics $[\mathrm{t}(4 ; 14), \mathrm{t}(14 ; 16)$ or $\operatorname{del}(17 \mathrm{p})]$ by FISH present & 48 & $1.18(1.06 ; 1.32)$ & 0.003 & $0.99(0.87 ; 1.13)$ & 0.875 \\
\hline \multicolumn{6}{|l|}{ ECOG Performance status } \\
\hline 0 & 111 & Reference & & Reference & \\
\hline 1 & 116 & $1.41(1.28 ; 1.54)$ & $<0.001$ & $1.41(1.27 ; 1.56)$ & $<0.001$ \\
\hline 2 & 45 & $1.25(1.09 ; 1.43)$ & 0.001 & $1.12(0.97 ; 1.30)$ & 0.129 \\
\hline 3 & 26 & $1.47(1.27 ; 1.69)$ & $<0.001$ & $1.40(1.17 ; 1.66)$ & $<0.001$ \\
\hline Best response to ongoing line of therapy & $\S$ & & & & \\
\hline sCR/CR/VGPR & 1050 & Reference & & Reference & \\
\hline $\mathrm{PR} / \mathrm{MR}$ & 1136 & $1.23(1.13 ; 1.35)$ & $<0.001$ & $1.45(1.29 ; 1.63)$ & $<0.001$ \\
\hline $\mathrm{SD} / \mathrm{PD}$ & 442 & $1.42(1.26 ; 1.60)$ & $<0.001$ & $1.46(1.24 ; 1.71)$ & $<0.001$ \\
\hline Number of prior lines of therapy & $\S$ & & & & \\
\hline 1 & 873 & Reference & & Reference & \\
\hline 2 & 519 & $0.95(0.85 ; 1.07)$ & 0.415 & $1.15(1.00 ; 1.33)$ & 0.051 \\
\hline 3 & 400 & $1.10(0.97 ; 1.26)$ & 0.131 & $1.27(1.07 ; 1.50)$ & 0.006 \\
\hline 4 & 314 & $1.30(1.13 ; 1.50)$ & $<0.001$ & $1.42(1.16 ; 1.74)$ & 0.001 \\
\hline 5 & 191 & $0.94(0.80 ; 1.12)$ & 0.513 & $0.91(0.72 ; 1.15)$ & 0.429 \\
\hline 6 & 212 & $1.60(1.35 ; 1.90)$ & $<0.001$ & $1.83(1.43 ; 2.33)$ & $<0.001$ \\
\hline $7+$ & 276 & $1.05(0.89 ; 1.23)$ & 0.566 & $1.45(1.14 ; 1.84)$ & 0.002 \\
\hline Ongoing line of therapy by regimen & $\S$ & & & & \\
\hline Melphalan $200 \mathrm{mg} / \mathrm{m}^{2}$ days $0-30$ & 434 & $1.19(1.04 ; 1.37)$ & 0.012 & $1.28(1.07 ; 1.53)$ & 0.006 \\
\hline IMID \pm steroid & 487 & Reference & & Reference & \\
\hline $\mathrm{PI} \pm$ steroid & 280 & $1.72(1.46 ; 2.02)$ & $<0.001$ & $1.80(1.50 ; 2.15)$ & $<0.001$ \\
\hline $\mathrm{CH} \pm$ steroid & 201 & $2.01(1.68 ; 2.40)$ & $<0.001$ & $1.79(1.48 ; 2.18)$ & $<0.001$ \\
\hline IMID + PI \pm steroid & 224 & $1.29(1.08 ; 1.53)$ & 0.004 & $1.19(0.99 ; 1.44)$ & 0.067 \\
\hline $\mathrm{PI}+\mathrm{CH} \pm$ steroid & 173 & $1.48(1.23 ; 1.78)$ & $<0.001$ & $0.94(0.76 ; 1.16)$ & 0.547 \\
\hline IMID $+\mathrm{CH} \pm$ steroid & 301 & $1.46(1.24 ; 1.70)$ & $<0.001$ & $1.14(0.95 ; 1.36)$ & 0.165 \\
\hline Daratumumab \pm steroid & 147 & $1.15(0.93 ; 1.42)$ & 0.192 & $1.29(1.02 ; 1.63)$ & 0.033 \\
\hline Daratumumab $+\mathrm{PI} \pm$ steroid & 37 & $0.60(0.41 ; 0.89)$ & 0.011 & $0.90(0.59 ; 1.37)$ & 0.624 \\
\hline Daratumumab + IMID \pm steroid & 171 & $1.26(1.04 ; 1.53)$ & 0.020 & $1.21(0.97 ; 1.51)$ & 0.094 \\
\hline Daratumumab + PI + IMID \pm steroid & 3 & $0.79(0.25 ; 2.45)$ & 0.677 & $0.85(0.26 ; 2.82)$ & 0.791 \\
\hline Five or more drugs & 294 & $2.18(1.87 ; 2.54)$ & $<0.001$ & $1.79(1.50 ; 2.14)$ & $<0.001$ \\
\hline Other & 33 & $2.31(1.50 ; 3.55)$ & $<0.001$ & $1.02(0.63 ; 1.63)$ & 0.949 \\
\hline Time from diagnosis & $\S$ & & & & \\
\hline 0-91 days after & 77 & Reference & & Reference & \\
\hline 92-183 days after & 472 & $0.53(0.45 ; 0.63)$ & $<0.001$ & $0.52(0.42 ; 0.65)$ & $<0.001$ \\
\hline $183-365$ days after & 278 & $0.61(0.51 ; 0.72)$ & $<0.001$ & $0.44(0.34 ; 0.56)$ & $<0.001$ \\
\hline 365-730 days after & 270 & $0.40(0.34 ; 0.47)$ & $<0.001$ & $0.26(0.20 ; 0.32)$ & $<0.001$ \\
\hline More than 730 days after & 404 & $0.26(0.23 ; 0.30)$ & $<0.001$ & $0.12(0.10 ; 0.16)$ & $<0.001$ \\
\hline Time from initiation of immunoglobulin replacement therapy & $\S$ & & & & \\
\hline Before & 524 & Reference & & Reference & \\
\hline $0-91$ days after & 335 & $2.34(2.02 ; 2.71)$ & $<0.001$ & $2.06(1.75 ; 2.43)$ & $<0.001$ \\
\hline 92-183 days after & 179 & $1.57(1.31 ; 1.88)$ & $<0.001$ & $1.26(1.02 ; 1.56)$ & 0.033 \\
\hline 184-365 days after & 249 & $2.39(2.03 ; 2.82)$ & $<0.001$ & $2.05(1.69 ; 2.48)$ & $<0.001$ \\
\hline Time from dates of progressive disease & $\S$ & & & & \\
\hline Before & 1315 & Reference & & Reference & \\
\hline $0-91$ days after & 281 & $1.13(0.98 ; 1.30)$ & 0.087 & $1.17(1.00 ; 1.37)$ & 0.051 \\
\hline $92-183$ days after & 33 & $0.95(0.65 ; 1.40)$ & 0.810 & $0.91(0.59 ; 1.41)$ & 0.682 \\
\hline $184-365$ days after & 20 & $0.61(0.37 ; 1.01)$ & 0.054 & $0.71(0.39 ; 1.29)$ & 0.260 \\
\hline
\end{tabular}

$\$$, the same patient is taken into account multiple times. $\mathrm{CH}$, chemotherapy (cyclophosphamide/low-dose melphalan/bendamustine/doxorubicine/liposomal doxorubicine/melflufen); CR, complete response; FISH, fluorescence in situ hybridisation; IMID, immunomodulatory agent (thalidomide/lenalidomide/pomalidomide); ISS, international staging system; sCR, stringent complete response; MR, minimal response; PD, progressive disease; PI, proteasome inhibitor (bortezomib/carfilzomib/ixazomib); PR, partial response; SD, stable disease; VGPR, very good partial response; WBLDCT, whole-body low-dose computer tomography. 